EPJ Web of Conferences 19, 09010 (2012)

DOI: $10.1051 /$ epjconf/20121909010

(C) Owned by the authors, published by EDP Sciences, 2012

\title{
Automated stellar spectra parameterisation for Gaia-RVS
}

\author{
G. Kordopatis ${ }^{1, a}$, A. Recio-Blanco ${ }^{1}$, P. de Laverny ${ }^{1}$, A. Bijaoui ${ }^{1}$, V. Hill ${ }^{1}$, \\ G. Gilmore ${ }^{2}$, R.F.G. Wyse ${ }^{3}$ and C. Ordenovic ${ }^{1}$ \\ ${ }^{1}$ Université de Nice Sophia Antipolis, CNRS, Observatoire de la Côte d'Azur, Cassiopée \\ UMR 6202, BP. 4229, 06304 Nice, France \\ ${ }^{2}$ Institute of Astronomy, University of Cambridge, Madingley Road, Cambridge CB3 OHA, UK \\ ${ }^{3}$ Johns Hopkins University, Baltimore, MD, USA
}

\begin{abstract}
Degeneracies in the stellar atmospheric parameters can pollute large spectroscopic surveys, introducing possible biases in the derived distances, kinematics and chemical abundances. We focus at the wavelength range shared by RAVE, the RVS of Gaia and the ESO-FLAMES/GIRAFFE spectrograph setups LR8 and HR21. Based on the algorithms DEGAS and MATISSE, trained on a grid of synthetic spectra, we propose a pipeline in order to derive the atmospheric parameters.
\end{abstract}

\section{INTRODUCTION}

Galactic archaeology aims to determine the evolution of the Galaxy from the chemical and kinematical properties of its stars. The analysis of current large spectroscopic surveys (thousands of stars) and future ones (millions of stars) require automated analysis techniques to obtain robust estimates of the stellar parameters. Several on-going and planned spetroscopic surveys have selected their wavelength region to contain the IR CaII triplet $(\lambda \sim 8500 \AA)$ and the work presented here focuses on the automatic analysis of such spectra. We developed and tested an automatic method which obtains estimates of the stellar atmospheric parameters (effective temperature, $T_{\text {eff }}$, surface gravity, $\log g$ and overall metallicity, $[\mathrm{M} / \mathrm{H}])$ from a stellar spectrum. We have also explored the degeneracies in parameter space, estimated the uncertainties in the derived parameter values and investigated the consequences of these limitations for achieving the goals of galactic archaeology [1].

\section{PRESENTATION OF THE METHOD}

We investigated two algorithms, both of which compare the observed spectrum to a grid of synthetic spectra, but each utilises a different mathematical approach to finding the optimum match and hence the best values of the stellar parameters. Our investigation of these algorithms robustness has wide applicability, since it amplifies the main problems that the other methods can encounter. The first algorithm, MATISSE [2], derives the values of each stellar parameter through a local fit to the spectrum such that each pixel in wavelength space is treated separately. The sensitivity of the flux at each wavelength to the value of a given stellar parameter is determined from the synthetic spectra. The observed spectrum is then projected using these sensitivity vectors to give an estimated value of the stellar parameters. This value depends on finding the true minimum in the fit and the algorithm must

\footnotetext{
ae-mail: Georges. Kordopatis@oca.eu
}

This is an Open Access article distributed under the terms of the Creative Commons Attribution-Noncommercial License 3.0, which permits unrestricted use, distribution, and reproduction in any noncommercial medium, provided the original work is properly cited. 

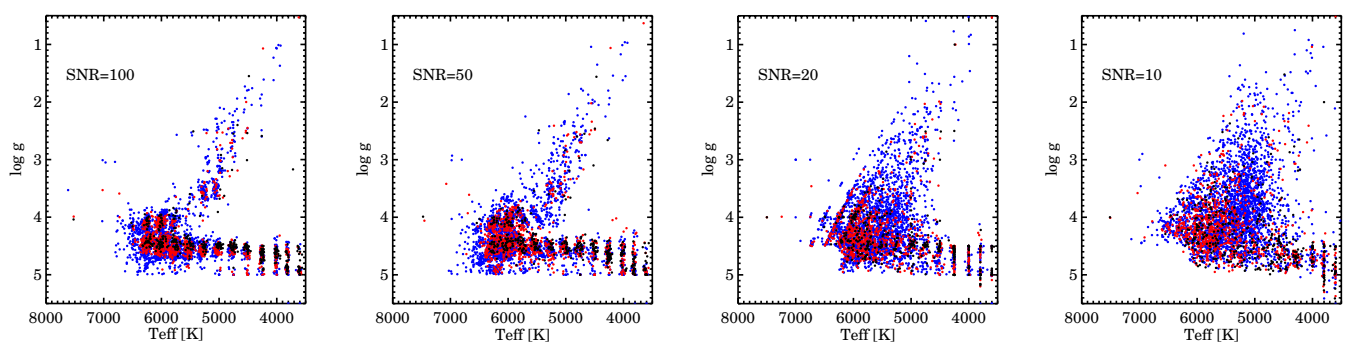

Figure 1. Dependance of the $\mathrm{H}-\mathrm{R}$ diagram for spectra analysed with the developed method, as a function of signal-to-noise ratio. The colour code, quantifies metallicity values (blue: $[\mathrm{M} / \mathrm{H}]<-0.75$, red: $-0.75<[\mathrm{M} / \mathrm{H}]<-$ 0.25 dex, black: $[\mathrm{M} / \mathrm{H}]>-0.25 \mathrm{dex})$.

avoid being trapped in false local minima. The second algorithm, DEGAS [3], uses a pattern-recognition approach and thus has a more global vision of the parameter space. The best-fit synthetic spectrum is derived through a series of comparisons between the observed and synthetic spectra, summed over wavelength pixels, with further refinements in the set of synthetic spectra after each stage i.e. a Decision Tree.

\section{VALIDATION OF THE METHOD: TESTS ON SYNTHETIC SPECTRA}

We computed $2 \times 10^{4}$ synthetic spectra, whose parameters follow an H-R diagram representative of the Milky Way stellar populations (obtained from the Besançon galactic model). The robustness of each method has then been tested for four different values of signal-to-noise (SNR 10, 20, 50, 100 pixel $^{-1}$ ). We identified physical degeneracies in different regions of the $\mathrm{H}-\mathrm{R}$ diagram: hot dwarf stars and giant stars share the same spectral signatures. Further, it is very difficult to determine an accurate value for the surface gravity of cooler dwarfs, because of the poor spectral signatures. These effects are intensified when the lack of information increases, such as happens for low-metallicity stars or spectra with low SNRs. Our results [1] demonstrate that the local projection method is preferred for spectra with high SNR, whereas the decision tree method is preferred for spectra of lower SNR. We therefore propose a hybrid approach, combining these methods, in order to iteratively normalise the spectra and obtain the stellar atmospheric parameters. We also demonstrate that sufficiently accurate results for the purposes of galactic archaeology studies are retrieved down to SNR 20 for typical parameter values of stars belonging to the local thin or thick disc, and for SNR down to $\sim 50$ for the more metal-poor giant stars of the halo (see Fig. 1).

\section{CONCLUSIONS}

Degeneracies in stellar parameters can introduce biases and systematic errors in derived quantities for target stars such as distances and full space motions. These can be minimised using the knowledge gained by thorough testing of the proposed stellar classification algorithm, leading to robust automated methods for the coming large spectroscopic surveys of stars in the Local Group. Tests done on synthetic and real data at $R \sim 6500$ and $\mathrm{SNR} \sim 50$, showed that accuracies for typical Galactic old thin disc stars, of $70 \mathrm{~K}, 0.12 \mathrm{dex}$ and $0.09 \mathrm{dex}$ for $T_{\text {eff }}, \log g$ and $[\mathrm{M} / \mathrm{H}]$ are achieved. As far the thick disc dwarfs are concerned, precisions of $108 \mathrm{~K}, 0.17 \mathrm{dex}$ and $0.12 \mathrm{dex}$ are reached. Finally, for typical halo giants, the method achieves accuracies of $94 \mathrm{~K}, 0.28 \mathrm{dex}, 0.17 \mathrm{dex}$. The proposed pipeline [1] has already been implemented in Java and has been delivered to the Gaia Data Processing and Analysis Consortium as one of the possible nodes of the whole processing pipeline. 
Assembling the Puzzle of the Milky Way

\section{References}

[1] Kordopatis G., Recio-Blanco A, de Laverny P., Bijaoui A., Hill V., Gilmore G., Wyse R.F.G., and Ordenovic C., A\&A, in press (2011)

[2] Recio-Blanco A., Bijaoui A., de Laverny P., MNRAS 370, (2006) 141

[3] Bijaoui A., Recio-Blanco A., de Laverny P., Ordenovic C., ADA6, (2010) 